\title{
Imaging patients with renal colic-consider ultrasound first
}

\author{
Carlos Nicolau • Michel Claudon • Lorenzo E. Derchi • E. Jane Adam • \\ Michael Bachmann Nielsen • Gerhard Mostbeck • Catherine M. Owens • \\ Christiane Nyhsen • Spyros Yarmenitis
}

Received: 24 October 2014 / Revised: 23 January 2015 / Accepted: 2 February 2015 / Published online: 21 May 2015

(C) The Author(s) 2015. This article is published with open access at Springerlink.com

\begin{abstract}
Renal colic is a common disease in Europe and a common cause of visit to the Emergency Department. Clinical diagnosis is usually confirmed by imaging modalities. Unenhanced computed tomography (CT) is considered the best diagnostic test due to its excellent accuracy detecting ureteral stones. However, ultrasound (US) should be considered as the primary imaging technique. It is a reproducible, non-invasive and non-expensive imaging technique, achieving accurate diagnosis in most cases without the need for radiation. Diagnosis is based on the presence of ureteral stones, but indirect findings such as the asymmetry or absence of ureteric jet, an increase of the resistive index or a colour Doppler twinkling artefact may help to suggest the diagnosis when the stone is not identified.
\end{abstract}

All authors are members of the ESR Working Group on Ultrasound

C. Nicolau $(\bowtie)$

Radiology Department, Hospital Clinic, Villarroel 170,

08036 Barcelona, Spain

e-mail: cnicolau@clinic.cat

M. Claudon

Children Hospital, University Hospital-Nancy Brabois, Rue du

Morvan, 54511 Vandoeuvre Les Nancy Cedex, France

L. E. Derchi

Emergency Radiology, DISSAL-Radiology, University of Genoa,

Genoa, Italy

E. J. Adam

St George's Hospital, Blackshaw Road, SW17 0QT London, UK

M. B. Nielsen

Rigshospitalet-Diagnostisk Center, Blegdamsvej 9,

2100 Copenhagen, Denmark
Main Messages

- Renal colic diagnosis is usually confirmed by imaging modalities.

- Imaging diagnosis of renal colic is based on the detection of ureteral stones.

- $C T$ is the most accurate imaging technique to identify ureteral stones.

- US allows correct diagnosis in most cases without using radiation.

- US should be used as the first imaging modality in patients with renal colic.

Keywords Computed Tomography $\cdot$ Hydronephrosis . Renal colic $\cdot$ Ultrasound $\cdot$ Ureteral calculi

G. Mostbeck

Department of Radiology, Wilhelminenspital, Montleartstr. 37,

1160 Vienna, Austria

C. M. Owens

Department of Radiology, Great Ormond Street, WC1N

3JH London, UK

C. Nyhsen

City Hospitals Sunderland FT, Kayll Road, Sunderland SR4 7TP, UK

S. Yarmenitis

Department of Radiology, Hygeia Hospital, 4, Erythrou Stavrou St, 15123 Maroussi, Greece 


\section{Introduction}

Renal stone disease is common in Europe, with a prevalence ranging between 2 and $8 \%$ [1]. It is a condition affecting relatively young individuals with an almost equal sex ratio and high tendency to recur: it is estimated that almost $50 \%$ of stone patients will present recurrence within 10 years [2]. Renal colics are secondary to ureteral obstruction by the stones. They are a common cause of visits to the Emergency Department and frequently require imaging evaluation [1, 2].

In most institutions non-enhanced multidetector computed tomography (MDCT) is considered the gold standard technique to evaluate these patients because of its accuracy in the detection of stones as well as of other pathological conditions mimicking renal colic [3]. It is also considered as the first imaging technique for the evaluation of patients with acute onset of flank pain by The American College of Radiology Appropriateness Criteria [4]. MDCT also allows an overall assessment of the 'stone load', which can help to plan the treatment. However, the vast majority of stones pass spontaneously, and CT imaging in the emergency department rarely alters immediate management [5]. Moreover, concerns about the over-utilisation of CT are growing because of increasing health care costs and, more importantly, exposure to ionising radiation $[6,7]$. The use of low-dose techniques can dramatically reduce the radiation dose [8], but these low-dose protocols have not been adopted worlwide [9]. On the other hand, ultrasound (US) is a safe, non-invasive and non-expensive technique able to evaluate patients with renal colic. However, its use remains controversial as it has good capability to identify dilatation of the excretory system even in non-experienced hands [10], but can have difficulties in directly demonstrating the stones, especially in the mid ureters, remaining operator dependent to detect stones and indirect findings that can help in the diagnosis. In addition, the absence of the 'indirect findings' does not exclude ureteral stones. In spite of these difficulties several papers, including a very recent multicentre comparative study between US and CT, have demonstrated the usefulness of US in the diagnosis and management of renal colic patients [11-13]

\section{When to use imaging in renal colic}

The diagnosis of renal colic is usually based on clinical grounds and immediate imaging is not always necessary [5, 14]. However, it is now common practice to perform imaging studies in all patients with suspected renal colic admitted to the Emergency Department. This may be due to fear of missing a life-threatening condition mimicking this condition, such as rupture of an aortic aneurysm, ovarian torsion or appendicitis, or to the need for imaging confirmation of the cause of symptoms before deciding on whether a patient may be discharged. At present, additional strong indications for imaging are the desire of patients to know the cause of their symptoms and the fear of litigation. If not in all patients, immediate imaging modalities are necessary in patients without clinical improvement after treatment, in cases with fever or leukocytosis, or in some special circumstances (i.e., patients with a single kidney and/or renal failure) [14, 15]; furthermore, imaging is also recommended in patients with remission of symptoms who do not eliminate the stone within a few days.

\section{CT}

CT has become the imaging study of choice for renal colic [2] because of its high sensitivity in the detection of renal and ureteral stones [16]. Moreover, when CT is performed with dual energy, it helps to characterise the composition of the renal stones. However, most hospitals do not have this technology, which in addition has very limited usefulness in case of ureteral stones. CT can identify the presence and size of stones with a very high accuracy of $>95 \%$ and is able to detect alternative diagnoses that simulate renal colic in 5-10\% of patients $[16,17]$. Nonetheless, in spite of its high accuracy, there is increasing concern about the increase of health care costs and radiation risk that accompanies CT scans, since the use of CT rarely changes the treatment plans of these patients [18]. In this way, in a recent retrospective study, Westphalen et al. determined the proportion of patient visits for flank or kidney pain receiving CT or US and calculated the diagnosis and hospitalisation rates for urolithiasis [19]. From 1996 to 2007, the use of CT to assess patients with suspected urolithiasis increased from 4.0 to $42.5 \%$ over the study period, and the use of US remained low, at about $5 \%$. However, the diagnosis of kidney stones, identification of significant alternate diagnoses or admission to the hospital did not increase.

The problem of exposure to radiation is very important in these patients, especially because of the possibility of cumulative radiation that is not usually well assessed when multiple CTs are performed in repetitive episodes of renal colic. Thus, the use of dedicated low-dose protocols is essential in these patients. In spite of the advances with dedicated low-dose CT protocols, a recent study evaluated renal colic CT studies conducted in 93 institutions in the USA from May 2011 to January 2013 [9] and demonstrated that reduced-dose renal CT protocols are used infrequently. The overall mean effective institutional dose was $11.2 \mathrm{mSv}$. Only $2 \%$ of the studies were conducted with a "reduced dose" of $3 \mathrm{mSv}$, and only $10 \%$ of institutions used an effective dose of $6 \mathrm{mSv}$ or less in at least $50 \%$ of patients. In another study performed in a single institution [6], the mean effective doses for a single study were $6.5 \mathrm{mSv}$ for SDCT and $8.5 \mathrm{mSv}$ for MDCT. Moreover, $4 \%$ of these patients (all with a known history of nephrolithiasis) underwent three or more studies, with estimated effective doses ranging from 19.5 to $153.7 \mathrm{mSv}$. 


\section{US}

Ultrasound is an accurate imaging technique to diagnose renal colic [11-13, 20, 21]. Moreover, this technique also allows the diagnosis of other renal diseases or extrarenal conditions that mimic renal colic (Table 1). The diagnosis of renal colic is based on the detection of stones and the consecutive obstruction of the excretory system (Fig. 1) [20]. Although the detection of dilatation of the excretory tract is very useful in the context of renal colic, this sign should be evaluated carefully, as dilatation does not necessarily mean obstruction, and the degree of dilatation does not reflect the severity of obstruction.

1. Features of stones in grey-scale and limitations.

Stones are identified as hyperechogenic foci with posterior shadowing. The most important limitations of US are the detection of small lithiases $(<5 \mathrm{~mm})$, which may be not recognised because of a partial volume effect or the absence of posterior shadowing, and the detection of stones not evaluable whatever the size in the mid ureters, which can be masked by overlying intestinal loops and gas, especially in obese patients. Regarding the detection of small intrarenal stones, one limitation is that hyperechoic foci can also be secondary to vascular or parenchymal calcifications, clots or arcuate arteries. The sensitivity of US in the detection of lithiasis varies greatly depending on the studies, with a wide range of sensitivities that usually depends on the size and location of the stones. Thus, Fowler described a very low sensitivity of $24 \%$ with $73 \%$ of calculi of $<3 \mathrm{~mm}$ not visualised.

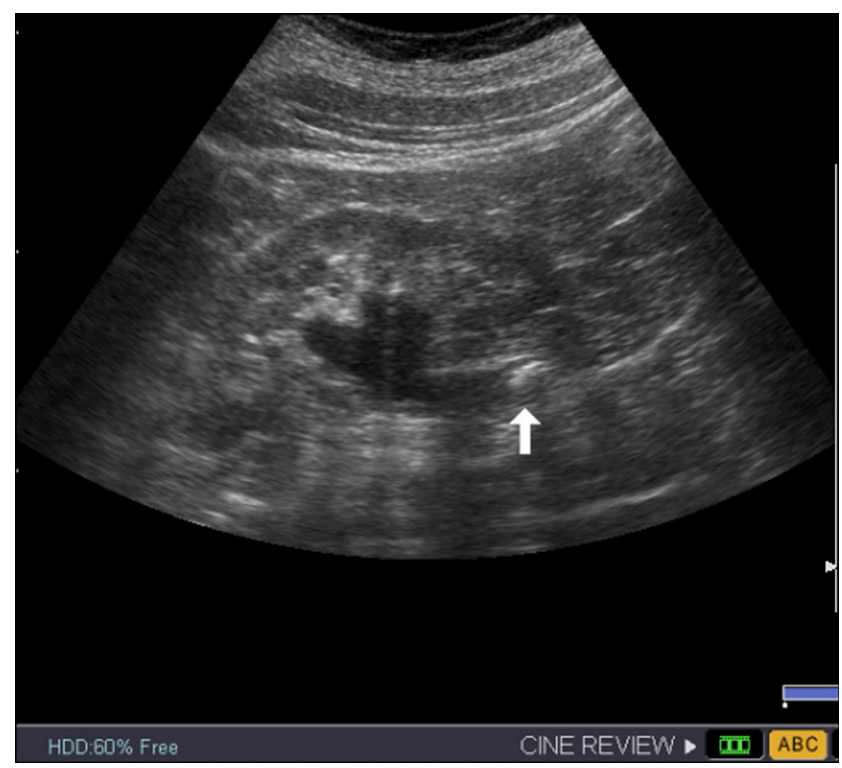

Fig. 1 Left proximal ureteral stone (arrow) producing hydronephrosis

Vallone described a sensitivity of $47.57 \%$ in the detection of renal lithiasis smaller than $5 \mathrm{~mm}$ [22], and Sheafor reported $61 \%$ sensitivity with $70 \%$ of calculi $\leq 3 \mathrm{~mm}$ not detected [23]. On the other hand, other studies have obtained very high sensitivities, such as $93 \%$ obtained in the study by Patlas performed by three experienced senior radiologists [21], $95 \%$ in the study of Dalla Palma [24] and $96 \%$ in the study of Middleton [12]. The specificity of US detecting ureteral calculi is $100 \%$ [23].

A complete study should include the kidney, ureterovesical joint (UVJ) and ureters. The presence of
Table 1 Alternative diagnoses in patients with renal colic

\author{
Entities \\ Pyelonephritis \\ Renal mass \\ Adnexal pathology: \\ Hemorrhagic ovarian cysts \\ Pelvic inflammatory disease \\ Endometriomas \\ Ovarian torsion \\ Ovarian neoplasms \\ Appendicitis \\ Diverticulitis
}

Dissection/ruptured aneurysms

\section{Most common US findings}

Mild disease may demonstrates no abnormality

Renal enlargement

Intra- or extrarenal fluid collections or abscesses may be present

Renal tumour (detection depends on tumour size)

Spontaneous subcapsular or perinephric bleeds may cause flank pain

\section{Heterogeneous cyst}

Thickened, dilated fallopian tube. Abscesses

Cyst with diffuse homogenous low-level internal echoes

Enlarged hypo or hyperechoic ovary with little or no intra-ovarian venous flow. In some cases twisted vascular pedicle is observed.

Ovarian masses

Noncompressible appendix with diameter $>6 \mathrm{~mm}$

Detection of diverticulum

Signs of inflammation of fat (dirty fat/stranding)

Thickened bowel wall $>4-5 \mathrm{~mm}$

Pericolic fluid or collections

Thin membrane fluttering in the aortic lumen

Dilatation of the aorta $>3 \mathrm{~cm}$, periaortic fluid collection 
UVJ oedema is considered a useful sign of a recent stone evacuation that can help to confirm the diagnosis of renal colic (Fig. 2). Regarding the detection of ureteral stones, the mid ureter is particularly difficult to identify,
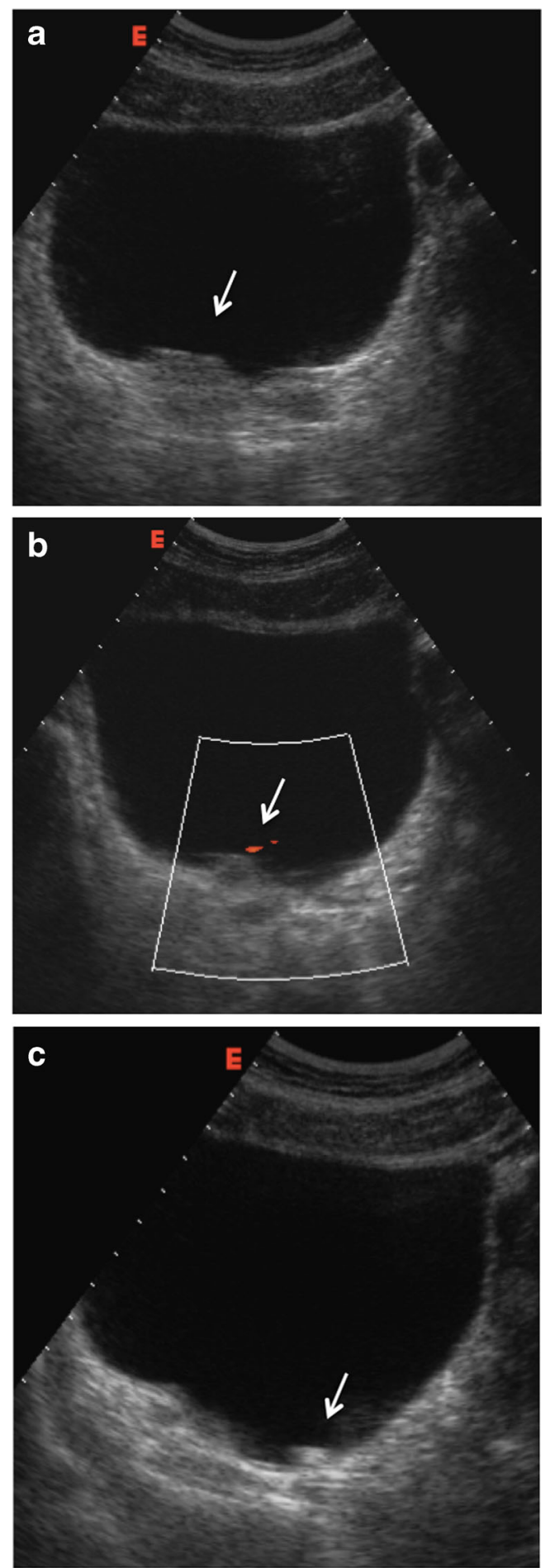

Fig. 2 Patient with right renal colic. a US image show an oedematous UVJ (arrow). b Colour Doppler shows the presence of a small ureteral jet confirming the patency of the ureter (arrow). c A small stone that moves according to patient decubitus is identified at the urinary bladder (arrow) especially in obese patients or because of interposition of the bowel. However, the visualisation of the mid ureter can be improved by compressing the area with the transducer or changing the patient's position (Fig. 3). The distal part of the ureter far to the vascular cross can also be very difficult to identify even with a well-dilated proximal ureter. In selected patients it can be helpful to perform transrectal or transvaginal US to evaluate the pelvic ureteral segment. Another limitation of US is that in the early phases of renal colic dilatation of the excretory tract is not always identified because there has not been enough time
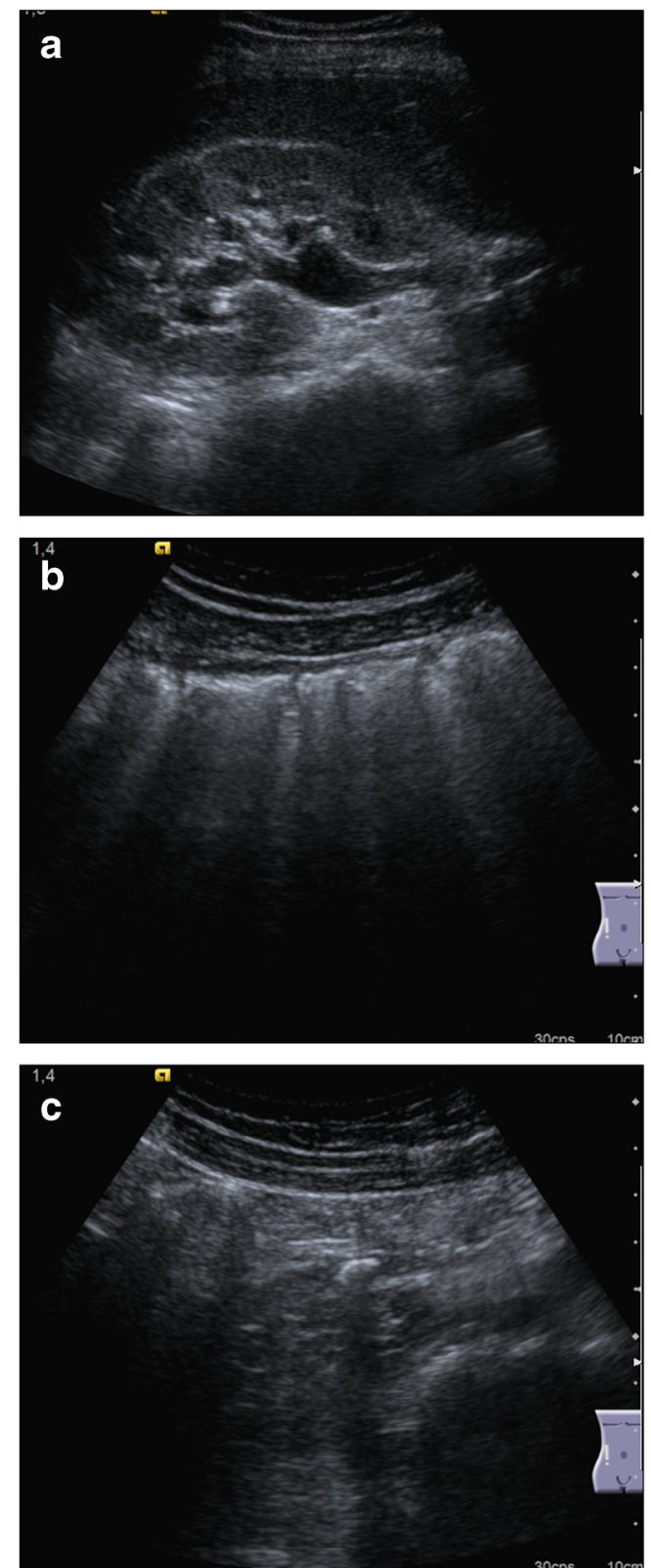

Fig. 3 a Right kidney hydronephrosis with ureteral dilatation. b Interposition of bowel loops that hampers the identification of the ureter. c Correct visualisation of a midureteral stone was obtained after compressing and displacing the bowel loops 
for its development; furthermore, dilatation can be minimal in case of small stones (Fig. 4) or can be absent in dehydrated patients. The US study should then be performed following hydration to ensure a distended urinary bladder (thus allowing a good acoustic window to the terminal ureter) as well as visibility of the ureteral jet (produced by the passage of urine from the ureter into the bladder), if there is not complete occlusion. The accuracy of diagnosing renal obstruction and stones improves with the use of Doppler US and colour Doppler identifying secondary signs.

2. Additional sonographic features that can help in the diagnosis.

- An absent, asymmetric and/or reduced ureteric jet from the ureteric orifices evaluated by colour Doppler is an additional indicator of obstruction. However, the presence of a positive ureteral jet does not rule out the presence of ureteral stones
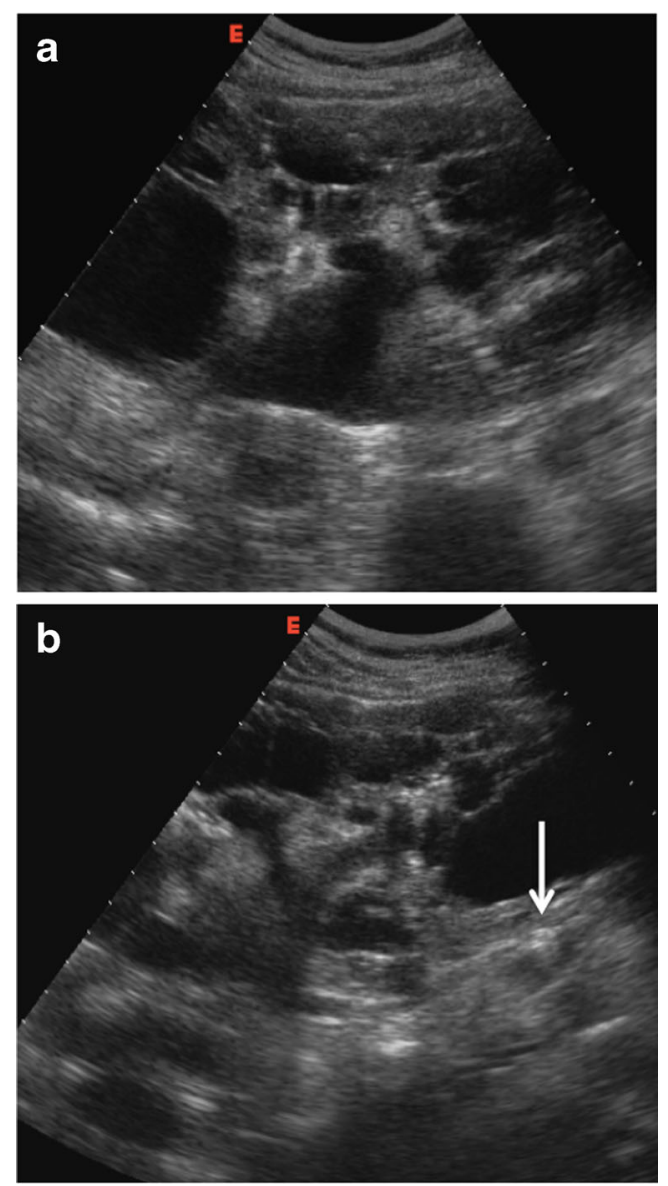

Fig. 4 Patient with known congenital polycystic kidney disease and mild renal impairment with flank pain and acute renal insufficiency. a Right kidney with multiple cysts and mild dilatation of the pelvis; $\mathbf{b}$ minimal dilatation of the ureter due to a small stone (arrow) at the upper third of the ureter
[23] since ureteral stones quite often only cause partial obstruction.

- Increased resistive index as a sign of acute obstruction, distinguishing between obstructive and nonobstructive dilatation [25]. A renal $\mathrm{RI}>0.70$ and/or a $10 \%$ difference between the kidneys is considered as diagnostic of obstructive uropathy [25].

- Colour Doppler twinkling artefact [15, 26, 27]. This artefact is a mixture of red and blue pixels on colour Doppler secondary to the "noise" produced from rough interfaces composed of sparse reflectors such as urinary stones. It is very useful to confirm findings of grey-scale, especially in doubtful cases due to small size of the stone [22] or located in difficult-to-visualise ureteral portions [27]. In the study by Moore et al. [15], the sensitivity of US improved from 47.6 to $86 \%$ when the twinkling sign was used. In the recent study by Ripolles et al., [27] which analysed the specific value of the twinkling artefact, the sensitivity of US using the twinkling artefact for detecting lithiasis was $90 \%$ and the specificity $100 \%$. A total of $78 \%$ of the lithiases showed the twinkling artefact, including three stones not identified by B-mode US, and in $68 \%$ of these stones, the artefact was detected before the stone itself with B-mode.

\section{Why use US first?}

The possibility of obtaining a diagnosis using US in patients with suspected renal colic has several advantages including its widespread availability and reduced cost over the use of CT [10]. Most importantly, the use of an algorithm in which US is used first can avoid radiation exposure in about $70 \%$ of cases [11, 20, 28]. Furthermore, as underlined by Catalano et al. and Ripolles et al., it seems safe to use this diagnostic technique even if it is known to have a lower sensitivity than CT in this field $[20,28]$. In fact, in both studies, spontaneous passage of the stone within a few days after the acute episode was observed in all patients with a false-negative US examination. In addition, a recent multicentre study clearly described the primary role of US in the investigation of renal colic today, demonstrating that the initial use of US is not associated with more complications, serious adverse events or hospitalisations than the initial evaluation with CT [13]. It must also be remembered that in patients in whom symptoms are not due to a renal colic US also has the ability to identify alternative diagnoses, albeit with a slightly lower sensitivity than CT, and that most of the important, life-threatening situations that may mimic renal colic can be recognised [29]. 
Nonetheless, US also has a few disadvantages: it may take longer to perform than nonenhanced $\mathrm{CT}$ and must be performed by an experienced examiner. The first of these limitations cannot be justified within the framework of the justification process of diagnostic studies using ionising radiation; radiologists are strongly advised to use alternative, nonionising techniques whenever possible. The second limitation may be difficult to overcome, especially if service is to be provided 24/24 h and 7/7 days. However, Dalla Palma et al. [24] have shown that high sensitivity results can be obtained in well-hydrated patients by general radiologists on call who are not specifically dedicated to US. The performance of US studies by radiologists also has an impact on the need for additional imaging techniques as demonstrated in the multicentre study of Smith-Bindman, in which $40.7 \%$ of the patients initially evaluated with US by emergency physicians, and only $27 \%$ of the patients initially evaluated by radiologists underwent additional CT [13].

Rethinking the imaging strategies in patients with suspected renal colic taking into account radiation protection considerations is possible and it has started both within the radiological community $[15,30]$ and among emergency physicians $[10,13]$. Urologists also agree on this topic: in the 2014 guidelines on urolithiasis of the European Association of Urology it is stated that in patients with renal stone disease US should be used as the primary procedure [31], and CT should be reserved for those patients who do not improve with conservative treatment or on suspicion of a nonurologic process [20].

\section{Conclusion}

Ultrasound can achieve a high sensitivity and specificity for the depiction of ureteral calculi and acute obstruction and has several advantages including its availability, lower cost and absence of radiation. Thus, it should be considered the first imaging modality in patients with renal colic. No more complications, serious adverse events, return emergency department visits or hospitalisations are expected using US first instead of CT. CT should be reserved for patients in whom US does not obtain a diagnosis, if symptoms do not resolve or there is a suspicion of alternative diagnoses.

Open Access This article is distributed under the terms of the Creative Commons Attribution License which permits any use, distribution, and reproduction in any medium, provided the original author(s) and the source are credited.

\section{References}

1. Johri N, Cooper B, Robertson W et al (2010) An update and practical guide to renal stone management. Nephron Clin Pract 116:c159-c171
2. Teichman JMH (2004) Clinical practice. Acute renal colic from ureteral calculus. N Engl J Med 350:684-693

3. Fulgham PF, Assimos DG, Pearle MS, Preminger GM (2013) Clinical effectiveness protocols for imaging in the management of ureteral calculous disease: AUA technology assessment. J Urol 189: 1203-1213

4. Coursey CA, Casalino DD, Remer EM et al (2012) ACR Appropriateness Criteria ${ }^{\circledR}$ acute onset flank pain-suspicion of stone disease. Ultrasound Q 28:227-233

5. Lindqvist K, Hellström M, Holmberg G et al (2006) Immediate versus deferred radiological investigation after acute renal colic: a prospective randomized study. Scand J Urol Nephrol 40:119-124

6. Katz SI, Saluja S, Brink JA, Forman HP (2006) Radiation dose associated with unenhanced CT for suspected renal colic: impact of repetitive studies. AJR Am J Roentgenol 186:1120-1124

7. Broder J, Bowen J, Lohr J et al (2007) Cumulative CT exposures in emergency department patients evaluated for suspected renal colic. J Emerg Med 33:161-168

8. McLaughlin PD, Murphy KP, Hayes SA et al (2014) Non-contrast $\mathrm{CT}$ at comparable dose to an abdominal radiograph in patients with acute renal colic; impact of iterative reconstruction on image quality and diagnostic performance. Insights Imaging 5:217-230

9. Lukasiewicz A, Bhargavan-Chatfield M, Coombs L et al (2014) Radiation dose index of renal colic protocol CT studies in the United States: a report from the American College of Radiology National Radiology Data Registry. Radiology 131601. doi:10.1148/ radiol.14131601

10. Dalziel PJ, Noble VE (2013) Bedside ultrasound and the assessment of renal colic: a review. Emerg Med J 30:3-8

11. Dalla Palma L, Stacul F, Mosconi E, Pozzi Mucelli R (2001) Ultrasonography plus direct radiography of the abdomen in the diagnosis of renal colic: still a valid approach? Radiol Med 102:222-225

12. Middleton WD, Dodds WJ, Lawson TL, Foley WD (1988) Renal calculi: sensitivity for detection with US. Radiology 167:239-244

13. Smith-Bindman R, Aubin C, Bailitz J et al (2014) Ultrasonography versus computed tomography for suspected nephrolithiasis. N Engl $\mathrm{J}$ Med 371:1100-1110

14. Bultitude M, Rees J (2012) Management of renal colic. BMJ 345: e5499

15. Moore CL, Scoutt L (2012) Sonography first for acute flank pain? J Ultrasound Med 31:1703-1711

16. Smith RC, Verga M, McCarthy S, Rosenfield AT (1996) Diagnosis of acute flank pain: value of unenhanced helical CT. AJR Am J Roentgenol 166:97-101

17. Pernet J, Abergel S, Parra J et al (2014) Prevalence of alternative diagnoses in patients with suspected uncomplicated renal colic undergoing computed tomography: a prospective study. CJEM 16:8-14

18. Zwank MD, Ho BM, Gresback D et al (2014) Does computed tomographic scan affect diagnosis and management of patients with suspected renal colic? Am J Emerg Med 32:367-370

19. Westphalen AC, Hsia RY, Maselli JH et al (2011) Radiological imaging of patients with suspected urinary tract stones: national trends, diagnoses, and predictors. Acad Emerg Med 18:699-707

20. Ripollés T, Agramunt M, Errando J et al (2004) Suspected ureteral colic: plain film and sonography vs unenhanced helical CT. A prospective study in 66 patients. Eur Radiol 14:129-136

21. Patlas M, Farkas A, Fisher D et al (2001) Ultrasound vs CT for the detection of ureteric stones in patients with renal colic. Br J Radiol 74:901-904

22. Vallone G, Napolitano G, Fonio P et al (2013) US detection of renal and ureteral calculi in patients with suspected renal colic. Crit Ultrasound J 5(Suppl 1):S3

23. Sheafor DH, Hertzberg BS, Freed KS et al (2000) Nonenhanced helical CT and US in the emergency evaluation of patients with renal colic: prospective comparison. Radiology 217:792-797 
24. Dalla Palma L, Stacul F, Bazzocchi M et al (1993) Ultrasonography and plain film versus intravenous urography in ureteric colic. Clin Radiol 47:333-336

25. Piazzese EMS, Mazzeo GI, Galipò S et al (2012) The renal resistive index as a predictor of acute hydronephrosis in patients with renal colic. J Ultrasound 15:239-246

26. Davran R (2009) The usefulness of color Doppler twinkling artifact in the diagnosis of urinary calculi. Eur J Radiol 71:378

27. Ripollés T, Martínez-Pérez MJ, Vizuete J et al (2013) Sonographic diagnosis of symptomatic ureteral calculi: usefulness of the twinkling artifact. Abdom Imaging 38:863-869
28. Catalano O, Nunziata A, Altei F, Siani A (2002) Suspected ureteral colic: primary helical CT versus selective helical CT after unenhanced radiography and sonography. AJR Am J Roentgenol 178:379-387

29. Van Randen A, Laméris W, van Es HW et al (2011) A comparison of the accuracy of ultrasound and computed tomography in common diagnoses causing acute abdominal pain. Eur Radiol 21:1535-1545

30. Patatas K, Panditaratne N, Wah TM et al (2012) Emergency department imaging protocol for suspected acute renal colic: re-evaluating our service. Br J Radiol 85:1118-1122

31. (2014) Guidelines on urolithiasis. European Association of Urology. In: http://www.uroweb.org/gls/pdf/22\%20Urolithiasis_LR.pdf. http://www.uroweb.org/gls/pockets/english/22Urolithiasis_LR.pdf. Accessed 4 May 2014 